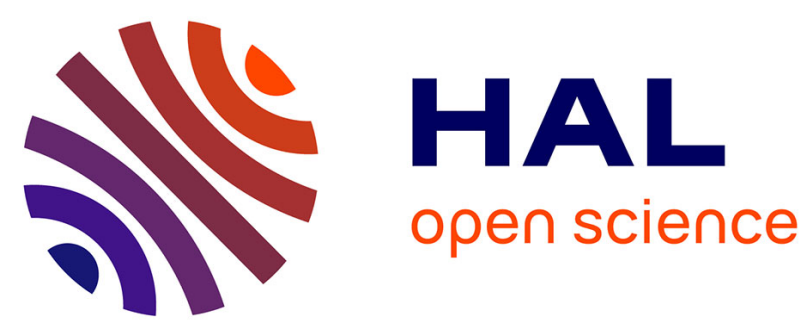

\title{
Global Instability in Shock Wave Laminar Boundary-Layer Interaction
}

Florian Guiho, Frédéric Alizard, Jean-Christophe Robinet

\section{To cite this version:}

Florian Guiho, Frédéric Alizard, Jean-Christophe Robinet. Global Instability in Shock Wave Laminar Boundary-Layer Interaction. Instability and Control of Massively Separated, Oct 2015, Prato, Italy. pp.97-102, 10.1007/978-3-319-06260-0_14 . hal-02570526

\section{HAL Id: hal-02570526 \\ https://hal.science/hal-02570526}

Submitted on 12 May 2020

HAL is a multi-disciplinary open access archive for the deposit and dissemination of scientific research documents, whether they are published or not. The documents may come from teaching and research institutions in France or abroad, or from public or private research centers.
L'archive ouverte pluridisciplinaire HAL, est destinée au dépôt et à la diffusion de documents scientifiques de niveau recherche, publiés ou non, émanant des établissements d'enseignement et de recherche français ou étrangers, des laboratoires publics ou privés. 


\title{
Global Instability in Shock Wave Laminar Boundary-Layer Interaction
}

\author{
F. Guiho, F. Alizard and J.-Ch. Robinet
}

\begin{abstract}
The linear global stability of an interaction between an oblique shock wave and a laminar boundary layer is carried out for various oblique shock angles. It is illustrated that such a flow acts as a noise amplifier. The least temporally damped global modes are classified into three main categories, low, medium and high frequencies. The high frequencies are localized into the attached boundary layer, the medium frequencies are associated with Kelvin-Helmholtz like structures along the shear layer and convective waves in the separated flow downstream whereas the low frequencies are driven by the interaction zone. In particular, a low frequency mode emerges which is scaled by the interaction length and the freestream velocity.
\end{abstract}

\section{Introduction}

Shock-wave/boundary-layer interactions (SWBLI) have important applications in a wide range of industrial problems, such as high speed flight occurring on aircraft, space/launch vehicles and projectiles. In particular, these interaction phenomena is a prior interest for supersonic/hypersonic flows where aerodynamic heating is a major factor [3]. The generation of shock waves by various surfaces of a vehicle or aircraft engine and the impingement of those shocks on other surfaces can greatly amplify the local heat transfer. When shock wave interacts with a laminar boundary layer, the experiments have shown that, for a sufficiently large pressure gradient amplitude, the boundary-layer becomes turbulent. It strongly modifies the interaction zone as well as its dynamics yielding to a reduced separated zone. Transition mechanisms are poorly known. However, in the presence of a low environmental noise or low upstream

F. Guiho · J.-Ch. Robinet ( $\varangle)$

DynFluid Laboratory, Arts et Métiers ParisTech, 151, Bd. de l'Hôpital, 75013 Paris, France

e-mail: Jean-christophe.ROBINET@ensam.eu

F. Guiho · F. Alizard

DynFluid Laboratory, CNAM, 151, Bd. de l'Hôpital, 75013 Paris, France 
disturbances, linear stability analysis can provide a good approximation of the position of laminar-turbulent transition (see for instance the $e^{N}$ method) [7]. In addition, when the interaction zone is higher, one may also trigger self-sustained low-frequency oscillations or three-dimensionality [6]. This specific dynamics can not be captured by a local linear stability analysis, a global analysis is thus required.

In this paper, the linear global stability of a two-dimensional interaction between an oblique shock wave and a laminar boundary-layer on a flat plate is carried out. In particular, it will be further examine whether such a flow may act as a resonator or a noise amplifier when it is subjected to two-dimensional perturbations. In addition, the global modes which are involved in the space-time dynamics will be highlighted.

\section{Governing Equations and Numerical Methods}

The present approaches are based on the standard small perturbation technique. The instantaneous flow is decomposed into base flow and unsteady perturbations:

$$
\mathbf{q}(x, y, t)=\mathbf{Q}_{b}(x, y)+\mathbf{q}^{\prime}(x, y, t)
$$

where $\mathbf{Q}_{b}=\left(\rho_{b}, \rho_{b} U_{b}, \rho_{b} V_{b}, \rho_{b} E_{b}\right)^{T}$. The decomposition (1) is introduced into compressible Navier-Stokes equations. The base flow $\mathbf{Q}_{b}$ is supposed to be an equilibrium solution of the 2-D compressible Navier-Stokes equations. The resulting equations are further simplified by considering that the perturbation is infinitesimal, i.e. the nonlinear fluctuating terms are neglected. Finally, compressible Navier-Stokes equations are transformed into a linear partial differential equations $\partial \mathbf{q}^{\prime} / \partial t=\mathscr{A} \mathbf{q}^{\prime}$ where $\mathbf{q}^{\prime}$ represents the conservative variables for the perturbation. Let-us introduce the following decomposition $\mathbf{q}^{\prime}=\hat{\mathbf{q}}_{k}(\mathbf{x}) e^{-i \Omega_{k} t}$ where the couple

leth. $\hat{\mathbf{q}}_{k}, \mathrm{f}_{\mathrm{k}}$ is the so-called global mode and is a solution of large eigenvalue probeigenfunction) and $\Omega_{k}$ (the complex circular frequency) respectively.

The numerical method implemented in CFD DynFluid PHOENIX solver is based on the finite-volume approach and on a cell centered discretization. Roe flux difference splitting scheme are employed to obtain advective fluxes at the cell interface. The MUSCL approach extends the spatial accuracy to third order. For viscous terms, a central difference method is used. For unsteady computations, the dual time stepping method is used, the derivative with respect to the physical time is discretized by a second-order formula. For more details about the numerical methods, see [4].

To obtain an equilibrium state of the compressible Navier-Stokes equations, the dual-time is turned off with a large CFL number with respect to the fully implicit method. 
(a)

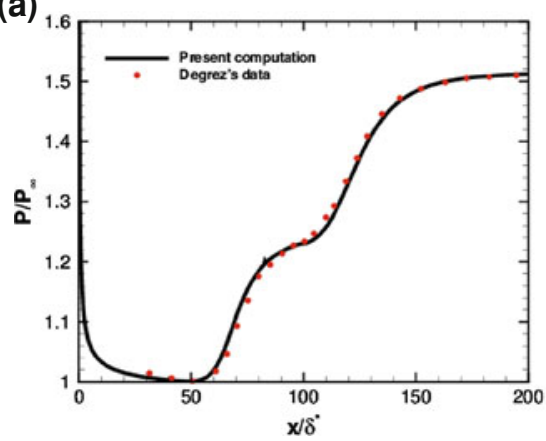

(b)

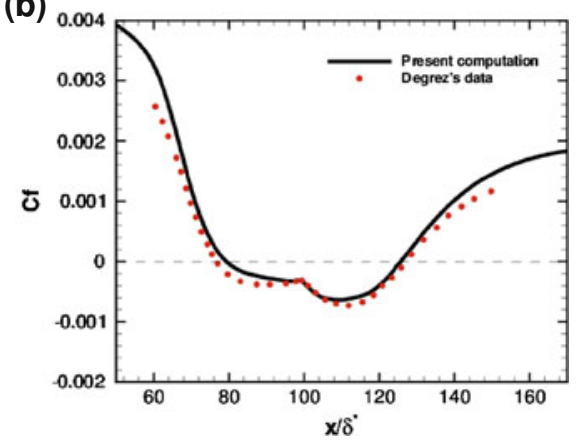

Fig. 1 Base flow characteristics for $\theta=30.8^{\circ}, R e_{\delta^{\star}}=886$. Comparison between numerical solution and Degrez's experiment a $P_{(x)} / P_{\infty} \mathbf{b} C_{f}(x)$

To perform a global stability analysis, the eigenvalues of the Jacobian matrix $\mathscr{A}$ are computed with a time-stepper approach, see [1]. The Arnoldi algorithm implemented into the temporal loop of PHOENIX code is used to obtain the most unstable modes, see [4].

\section{Base Flow}

The configuration studied is similar as the case of Degrez et al. described in [2]. An oblique shock wave, whose the angle of the shock relative to the horizontal axis is referred as $\theta$ hereafter, is made to impinge on the flat plate laminar boundary layer at $M_{\infty}=2.15$ by enforcing at the inlet boundary discontinuous conditions that satisfy the Rankine-Hugoniot relations. In addition, the stagnation pressure is fixed at $12,300 \mathrm{~Pa}$. Nonreflecting boundary conditions are enforced at the freestream and downstream boundaries. Finally, Dirichlet boundary conditions for both the velocity components and the temperature are prescribed at the adiabatic wall.

A preliminary computation is performed by considering a laminar boundary layer without impinging shock. Hence, $\delta^{*}$ is the displacement thickness taken at the theoretical location where the shock impinges the boundary layer, $U_{\infty}, \rho_{\infty}$ and $\mu_{\infty}$, are the velocity, the density and the molecular viscosity at the freestream.In the next, the Reynolds number based on the previous quantities is fixed to $R e_{\delta^{*}}=886$. After having reached a stationary state for the laminar boundary layer, the shock is introduced at the inflow condition. Hereafter, we consider four-different values for $\theta=30.8^{\circ}, 31.2^{\circ}, 31.6^{\circ}$ and $32^{\circ}$ while holding the Reynolds number and the Mach number constants. Finally, we note $L_{\text {sep }}$ and $L_{\text {int }}$ the separation and the interaction length respectively.

The computational domain $\mathscr{D}$ extends from $\left[x_{0} / \delta^{\star} ; x_{n} / \delta^{\star}\right] \times\left[y_{0} / \delta^{\star} ; y_{n} / \delta^{\star}\right]=$ $[0 ; 400] \times[0 ; 250]$ in the streamwise and wall-normal directions respectively. Figure 1a, b show comparisons between the base flow obtained by the PHOENIX 


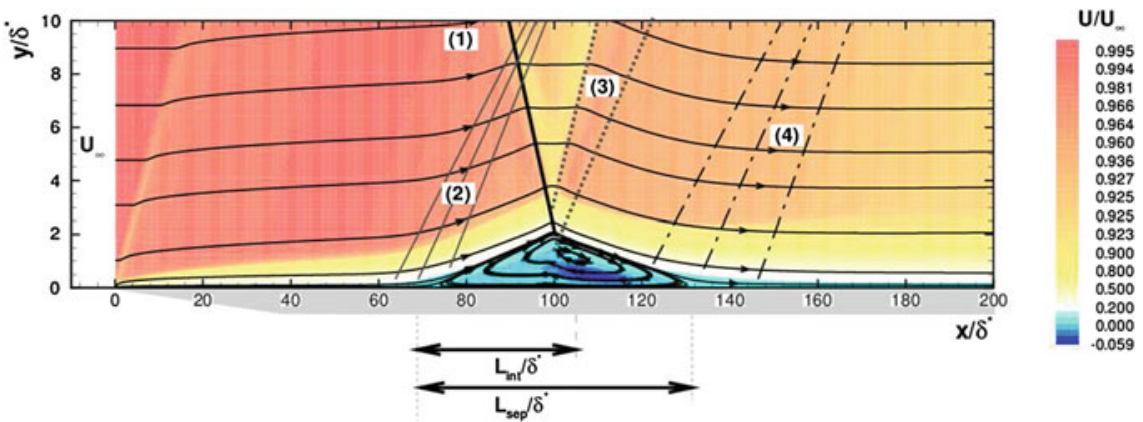

Fig. 2 Base flow characteristics for $\theta=30.8^{\circ}, R e_{\delta^{\star}}=886$. Iso-lines of streamwise velocity $U(x, y)$, streamlines and some characteristic phenomena: $\mathbf{1}$ incident shockwave; $\mathbf{2}$ separated waves; 3 expansion waves; $\mathbf{4}$ reattachment waves

Fig. 3 Eigenspectrum for Degrez's case, $\theta=$ $30.8^{\circ}$ and $R e_{\delta^{\star}}=886 . S t=$ $\Omega_{r} L_{i n t} /\left(2 \pi U_{\infty}\right)$

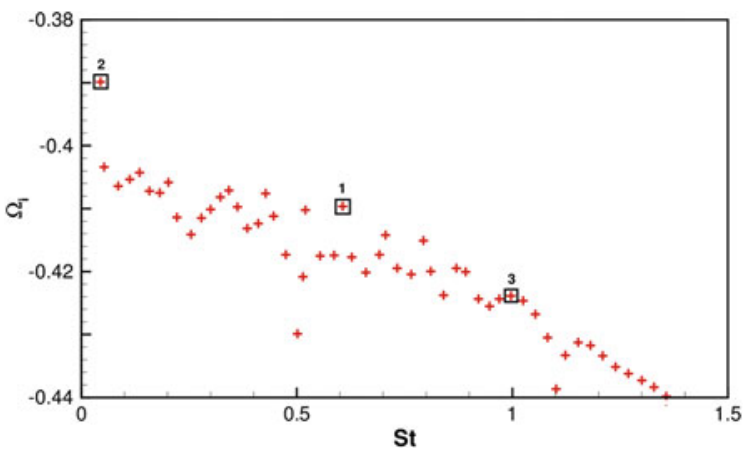

code and data from the experiments of Degrez et al. [2] for $\theta=30.8^{\circ}$. Both the wall pressure distribution (Fig. 1a) and the friction coefficient (Fig. 1b) exhibit a good agreement with the experimental results. As an example, we plot the streamwise velocity for $\theta=30.8$ in Fig. 2.

\section{Global Stability Analysis}

\subsection{Stability for the Shock Wave Angle $\theta=30.8$}

As shown in Fig. 3, all the global modes are damped temporally which is consistent with the experiment of Degrez et al. [2] where none global unsteadiness is observed. Such a flow acts therefore as a noise amplifier.

The modes labelled 1,2,3, medium, low and high frequencies respectively, are shown in Fig. 4a, b and c. All these modes are associated with convective waves which are travelling along the shear layer in the separated zone and the attached boundary 


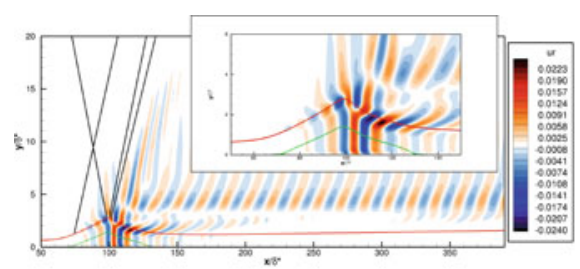

(a) Mode labelled 1 in Fig. 3.

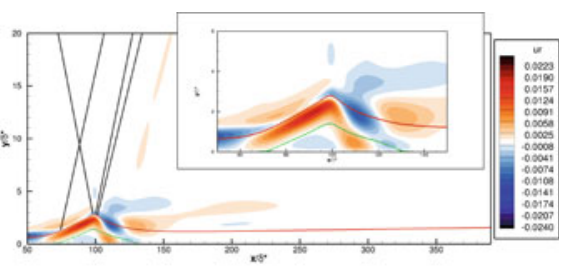

(b) Mode labelled 2

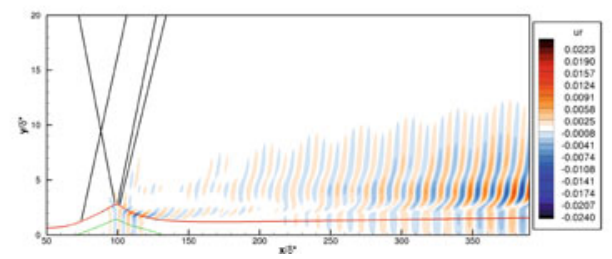

(c) Mode labelled 3

Fig. 4 Eigenfunctions for $\theta=30.8^{\circ}, R e_{\delta^{\star}}=886$. The streamwise component is shown

layer. In particular, lower the frequency is, more the spatial support of the global mode is localized inside the interaction region. Finally, the medium frequencies are clearly associated with Kelvin-Helmholtz like structures along the shear layer which emits Mach wave radiation in the vicinity of the impingement of the incident shock.

One may precise that the convergence of our results have been carefully checked by carrying out the global stability analysis with a large computational box which extends up to $400 \delta^{\star}$ and a coarse mesh, where 20 grid points per wavelength are used for the mode labelled 3 for instance.

\subsection{Influence of the Shock Wave Angle}

When the incident angle increases, the interaction becomes stronger, causing an increase in the recirculation zone (not shown here for the sake of conciseness). The spectrum for $\theta=30.8,31.2,31.6$ and 32 are shown in Fig. 5. Because all the modes are damped temporally for all the shock angles which are considered, the present shock wave-laminar boundary layer interaction is clearly identified as a noise amplifier. Hence, our global stability analysis further confirms the DNS results of Pagella et al. [5]. In addition, it is interesting to remark that a low frequency mode emerges for all equilibrium states (labelled as 2 in Fig. 3). When rescaled by the interaction length $L_{i n t}$ and the freestream velocity $U_{\infty}$, the corresponding frequency is seen to be almost constant around $S_{t} \approx 0.05$. 


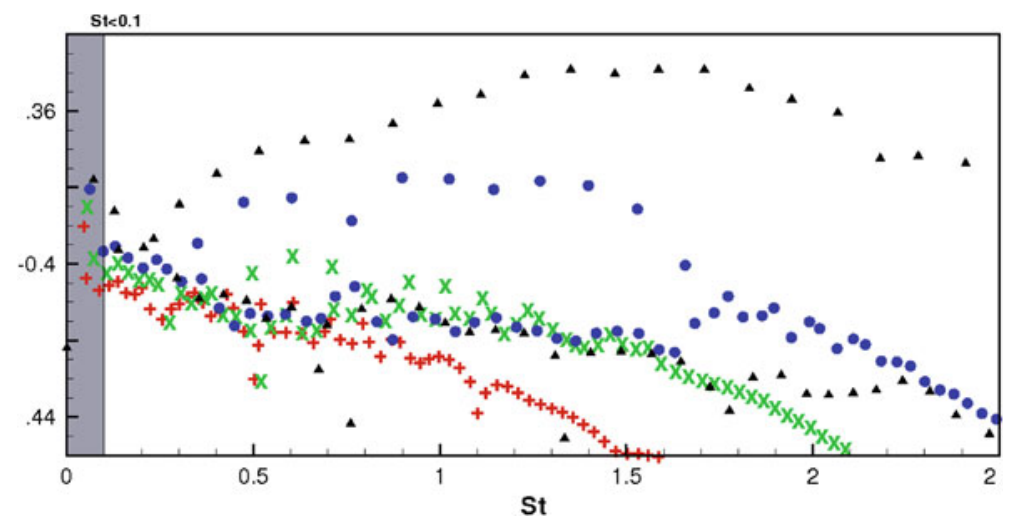

Fig. 5 Eigenspectrum for various shock angles $\theta\left(30.8^{\circ}(+), 31.2^{\circ}(\times), 31.6^{\circ}(\bullet), 32.0^{\circ}(\mathbf{\Lambda})\right] \cdot R e_{\delta^{\star}}=$ 886

\section{Conclusion}

The linear global stability analysis of the interaction between an oblique shock wave and a laminar boundary-layer confirms that such a flow acts as a noise amplifier, even for large shock wave angles. The global modes exhibit a Kelvin-Helmholtz like structure developing in the shear-layer. The lower frequency modes are mainly localized in the interaction zone. Finally, a low frequency mode strongly localized in the interaction zone and scaled by the interaction length and the freestream velocity emerges which may provides new insight about the low frequencies unsteadiness observed in the turbulent regime.

\section{References}

1. Bagheri S, Akervik E, Brandt L, Henningson DS (2009) Matrix-free methods for the stability and control of boundary layer. AIAA J 47(5):1057-1068

2. Degrez G, Boccadoro CH, Wendt JF (1987) The interaction of an oblique shock wave with a laminar boundary layer revisited. an experimental and numerical study. J Fluid Mech 177: 247-263.

3. Délery J, Marvin JG (1986) Shock-wave boundary layer interactions. Technical report, AGARDograph

4. Guiho F, Alizard F, Robinet J-C (2013) Global stability analysis with compressible cfd solver. AIAA Paper-2013-2620.

5. Pagella A, Rist U, Wagner S (2002) Numerical investigations of small-amplitude disturbances in a boundary layer impinging shock wave at ma $=4.8$. Phys Fluids 14(7):2088-2101

6. Robinet J-Ch (2007) Bifurcations in shock wave / laminar boundary layer interaction: global instability approach. J Fluid Mech 578:67-94

7. Tullio N, Sandham ND (2012) Transitional shock-wave/boundary layer interaction behind a roughness element. In: Kontis K (ed) 28th International symposium on shock waves. Springer, Heidelberg, pp 439-445 